\title{
Cervical intra-epithelial neoplasia in HIV-positive women after excision of the transformation zone - does the grade change?
}

\author{
Firdousi Kabir, Cyril van Gelderen, James McIntyre, Pam Michelow, Dianne Turton, Yasmin Adam
}

Objective. After previously reporting the presence of disease by cytology findings after treatment for cervical intra-epithelial neoplasia (CIN) in $64.6 \%$ of HIV-infected women and in $13.0 \%$ of HIV-negative women, we aimed to determine the severity of cytological disease after treatment in HIV-infected women.

Methods. We studied HIV-infected $(N=571)$ women treated at the Colposcopy Clinic at Chris Hani Baragwanath Hospital, Gauteng, between April 2003 and December 2006. We compared the initial histology results with Pap smears $\geq 6$ months later, and evaluated factors associated with reduction in the grade of disease.

Results. Mean age was 36.68 (SD+7.33) years; mean parity was 2 (SD+1.46); mean CD4+ count was 242.70 cells/ $\mu \mathrm{l}(\mathrm{SD}+187.56)$; $262(45.80 \%)$ were receiving antiretroviral treatment. Persistent disease was detected on the repeat Pap smear in 199 (65.03\%); of these, $223(72.88 \%)$ were of a lesser grade than in the original histology results. Of the 152 with histologically confirmed CIN3, 67 (44.08\%) had improved to a lesser grade, and $54(44.63 \%)$ had normal cytology results. Among the latter two subject groups ( $n=141)$ who had CIN2 histologically, 91 (64.53\%) had improved, 29 (20.57\%) remained unchanged, and 20 (14.88\%) had CIN3; 13 (4.25\%) patients with CIN1 returned for follow-up; 11 (84.62\%) of these had normal Pap smears and 2 (15.38\%) had CIN3.

Conclusion. Recurrences were of a lesser degree than initial histology results. This reduction in the grade of disease was related to CD4+ count, complete excision and parity. Antiretroviral therapy use did not improve outcome, perhaps owing to low initial CD4 counts.

S Afr Med J 2012;102(9):757-760. DOI:10.7196/SAMJ.5050
South Africa (SA) has a dual burden of cancer of the cervix and HIV infection. ${ }^{1,2}$ The prevalence of abnormal Papanicolaou (Pap) smears has been found to be up to $75 \%$ in HIV-positive women in SubSaharan Africa. ${ }^{3}$ Pre-malignant disease of the cervix is of a higher grade and progresses more rapidly in HIV-infected women.

Adequate screening and appropriate treatment reduces the incidence of cervical cancer in developed countries. ${ }^{4}$ The national screening programme for the prevention of cervical cancer in SA started in 2001 and was based on data pertaining to HIV-negative women. However, in patients treated for cervical intra-epithelial neoplasia (CIN), the risk of developing cancer is still 2.8 times greater than in the general population, and may be more in women with recurrent disease. ${ }^{5}$ Studies show that the recurrence of CIN after treatment was between $20 \%$ and $65 \%$ in HIV-infected women. ${ }^{67}$

We aimed to assess whether the cervical disease that occurs in HIV-infected women after treatment is of a different grade of disease compared with the histology results at presentation.

Department of Obstetrics and Gynaecology, Chris Hani Baragwanath Academic Hospital and University of the Witwatersrand, Johannesburg

Firdousi Akhter Kabir, MB BS, BSc (Hons), FCOG (SA)

Cyril Jack van Gelderen, MB ChB, FRCOG, FCOG (SA)

Yasmin Adam, BSc, MB BCh, FCOG (SA), MSc (Biostatistics \& Epidemiology)

Anova Health Institute, Johannesburg, and School of Public Health, University of Cape Town

James Alasdair McIntyre, MB ChB, FRCOG

Department of Anatomical Pathology, Faculty of Health Sciences, University of the Witwatersrand, and National Health Laboratory Services, Johannesburg

Pam Michelow, MB BCh, MSc

Diane Turton, MB BCh, FCPath (SA)

\section{Objectives}

We aimed to determine the grade of cervical pathology as reported cytologically in HIV-infected women at least 6 months after local excision compared with the histology findings at the time of treatment, and to assess the factors associated with a lesser grade of disease at follow-up.

\section{Materials and methods}

This was a retrospective cross-sectional study, using records of all HIV-positive patients who were treated at the Colposcopy Clinic at Chris Hani Baragwanath Academic Hospital (CHBAH) between April 2003 and December 2006. Follow-up data were extracted until December 2008. HIV status was ascertained by self-report. When the HIV test was performed more than 6 months before the visit and was negative, it was recorded as unknown in the database. Voluntary counselling and testing was only offered from 2006.

Women are referred to the Colposcopy Clinic from approximately 22 clinics in the Johannesburg metropolitan area. HIV-positive women with any abnormal cytology are referred. The Colposcopy Clinic is a 'see-and-treat' service where patients are offered immediate treatment at the time of the colposcopic diagnosis. Colposcopies are performed or supervised by experienced colposcopists. In view of the uncertainty of the natural history in HIV-infected women, our management in 2006 was more aggressive, and they were treated with large loop excision of the transformation zone (LLETZ) even when colposcopy suggested CIN1. HIV-negative women are treated with local excision when the Pap smear and colposcopy suggest CIN2 or more severe grades of disease.

HIV-positive women treated with excision of the transformation zone, using LLETZ or cone biopsy, formed the study group. Women with normal histology or invasive carcinoma on histology, or who had undergone hysterectomy or a second treatment, were excluded.

Pap smears were reported according to the Bethesda classification, ${ }^{8}$ which allows additional descriptors of CIN1, 2 and 3 . We are aware of the cytological limitations in differentiating CIN2 and CIN3 but this aids in the triage of colposcopy clinic patients. The CIN terminology was used for direct comparison between cytology and histology. 
Follow-up is by Pap smear at 6 or 12 months after treatment, depending on the histology. However, some patients delay their return for their own reasons, and the maximum time between treatment and follow-up was 60 months. Any abnormal Pap smear at any time during the follow-up was used in the analysis. If there was more than one abnormal Pap smear, the worst grade was used in the analysis. We did not consider the Pap smear after a second treatment.

The research was approved by the Human Research Ethics committee of the University of the Witwatersrand (ref. M090336).

Data were extracted from the colposcopy database where patient information is prospectively recorded. All analysis was done using STATA 10 statistical software (Stata Corp, Texas, USA).

The referral Pap smears, demographic factors and histology results were described using frequencies for categorical variables, and means and medians for continuous variables. We then compared the histology results obtained at the time of initial treatment with Pap smear results performed at least 6 months later in those women who came back for follow-up. For the univariate analysis, the outcome we used was any reduction in the grade of disease or a normal Pap smear as the outcome. All Pap smears that were normal (CIN2 or less when the original histology was CIN3; or CIN1 when the original histology was CIN2) were classified as 'lesser grade of disease'. A logistic regression analysis was performed to evaluate factors associated with a lesser degree in the grade of disease or normal cytology results on Pap smear 6 months after treatment. Factors evaluated for association were age, parity, $\mathrm{CD} 4+$ count and the use of antiretroviral treatment (ART). A multivariate logistic regression analysis for all variables with a $p$-value $<0.20$ was also performed (results not shown). The assumption of linearity in the logit scale was also assessed and the Hosmer- Lemeshow goodness-of-fit test was used for model diagnostics.

In view of the large loss to follow-up, an analysis comparing the women who were lost to follow-up with those who attended was performed. Categorical variables were compared using the chisquared test; for differences in means, the $t$-test was used.

\section{Results}

A total of 1384 patients attended the Colposcopy Clinic between April 2003 and December 2006. Of these, 322 (23.27\%) of unknown HIV status and 417 (30.13\%) HIV-negative women were excluded; 645 (46.60\%) patients were HIV-positive (Fig. 1). The mean age was 36.68 years $(\mathrm{SD}+7.33)$, with a range of $23-59$. The mean parity was 2 (SD+1.46) with a range of $0-9$.

The mean CD4+ was 242.70 (SD+187.56). The range of CD4+ counts was between 4 and 1222 cells/ $\mu$ l. More than half of the

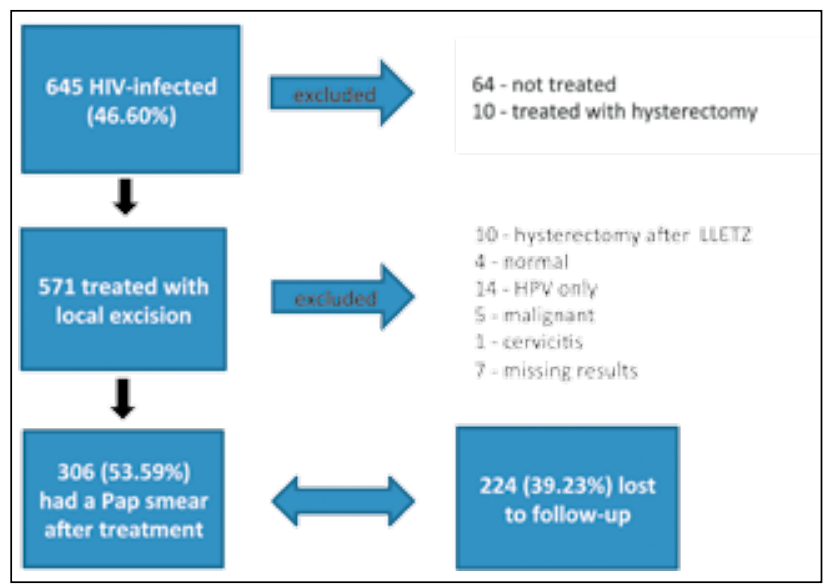

Fig. 1. Flow chart showing study sample. women were severely immunocompromised; 263 (52.08\%) patients had a CD4+ count equal to or less than 200 cells $/ \mu l$. The CD4+ was not recorded in 71 patients. Viral load is not performed routinely in this clinic. Of the 571 women, 262 (45.88\%) were on ART at the first visit to the Colposcopy Clinic; most were being treated in the public health service and were on AZT, 3TC and nevirapine/efavirenz. We were unable to assess for how long the women had been on ART. Table 1 shows Pap smear abnormalities at referral

The following colposcopic diagnoses were made: 41 (7.18\%) CIN1, 234 (40.98\%) CIN2, 244 (42.73\%) CIN3, 22 (3.85\%) were suggestive of invasive or micro-invasive disease, $2(0.35 \%)$ were normal, $1(0.18 \%)$ condyloma; and data had not been recorded in 27 (4.73\%).

There were 19 (3.30\%) women with normal histology results, cervicitis or HPV only on the histology findings (Table 2). The number of women with malignancy was less than $1 \%$, which is similar to other colposcopy clinics. ${ }^{9}$

In patients where a histology report could not be found, repeat colposcopy was performed and treatment offered, depending on the colposcopic findings. These findings were not analysed further.

The presence of disease at the margins of excision is an important predictor of recurrent or persistent disease in patients with CIN. ${ }^{10}$ The affected area was reported as being completely excised in 207 $(38.33 \%)$ subjects, the lesion was present at the ectocervical margin in 211 (39.55\%), and at the endocervical margin in 45 (8.38\%) while both margins were involved in $68(12.66 \%)$. The margin status was not reported by the laboratory in $6(1.12 \%)$ patients, and was not recorded in the database in a further $3(0.50 \%)$.

Table 1. Description of Pap smear abnormalities at referral

\begin{tabular}{ll}
\hline Pap smear at referral & N=571 (\%) \\
\hline High-grade squamous intra-epithelial lesion & $463(81.09)$ \\
Low-grade squamous intra-epithelial lesion & $63(11.03)$ \\
Atypical squamous cells of undetermined significance & $9(1.58)$ \\
Atypical squamous cells cannot exclude a high-grade & \\
lesion & $26(4.55)$ \\
Atypical glandular cells & $1(0.18)$ \\
Malignant cells/cannot exclude malignancy & $6(1.05)$ \\
Normal/clinical suspicion* & $1(0.18)$ \\
Missing cytology/came with a histology result ${ }^{\dagger}$ & $2(0.35)$ \\
& $*$ Where the Pap smear is reported as normal, but the cervix is clinically abnormal. \\
'Some women had had a biopsy for a clinically abnormal cervix, and did not have a Pap \\
smear.
\end{tabular}

Table 2. Description of histology results after local excision of the transformation zone

\begin{tabular}{ll}
\hline Histology on biopsy, cone or LLETZ & $\mathbf{N = 5 7 1 ( \% )}$ \\
\hline Cervical intra-epithelial neoplasia 1 & $49(8.58)$ \\
Cervical intra-epithelial neoplasia 2 & $226(39.58)$ \\
Cervical intra-epithelial neoplasia 3 & $265(46.41)$ \\
HPV only & $14(2.45)$ \\
Invasive carcinoma & $5(0.88)$ \\
Cervicitis & $1(0.18)$ \\
Normal & $4(0.70)$ \\
Missing data & $7(1.23)$
\end{tabular}




\section{Pap smears results at follow-up}

The overall number of women with any abnormal Pap smear during the follow-up period was 199 (65.03\%), and 107 (34.97\%) were reported as being negative for intra-epithelial lesion or malignancy. None had progressed to invasive disease during the follow-up period.

Fig. 2 shows the original histology results and the Pap smear result at follow-up. Of the women $(n=199(65.03 \%))$ with an abnormal Pap smear at follow-up, most of the abnormalities were of a lesser grade; $111(36.27 \%)$ had cytological abnormalities that required repeat treatment (HSIL-109, ASC-H-1, AGC-1). The remainder (195 (63.73\%)) had a LSIL and were followed up with Pap smears. Initial management of LSIL included LLETZ because we were uncertain as to the rate of progress in women who were HIV infected. However, it became apparent at follow-up that progression was infrequent, and therefore continued observation with repeat Pap smears was a reasonable follow-up regimen.

Factors associated with cytology results which were of a 'lesser grade than the original histology' or 'normal' at follow-up

Factors associated with lesser grade or normal cytology are shown in Table 3. A parity of $1-2$ was associated with twice the chance of having a lesser grade of disease compared with women with no pregnancies. The use of ART reduced the chance of a 'lesser grade of disease' and this association remained in the multivariate analysis when controlling for CD4+ count (results not shown). However, we

Table 3. Univariate analysis of factors associated with a lesser grade of disease

\begin{tabular}{lll}
\hline Variables & Univariate OR $(95 \%$ CI $)$ & $p$-value \\
\hline Age $>30$ & $1.09(0.54-2.15)$ & 0.81 \\
Parity: referent nulliparity & & \\
$\quad$ Parity-1 - & $2.19(1.06-4.49)$ & 0.03 \\
$\quad$ Parity $>3$ & $1.77(0.79-3.91)$ & 0.15 \\
ARV use & $0.50(0.30-0.82)$ & 0.00 \\
CD4 category: Referent: $<200$ & & \\
$\quad$ CD4 200 - 350 & $2.50(1.08-5.71)$ & 0.03 \\
CD4 350 - 500 & $1.28(0.49-3.28)$ & 0.61 \\
Margins referent: clear margins & & 0.03 \\
Both margins involved & $0.41(0.18-0.92)$ & 0.04 \\
Ectocervical margin & $0.54(0.30-0.99)$ & 0.95 \\
Endocervical margin & $0.96(0.33-2.84)$ &
\end{tabular}

were unable to assess the length of time that patients were on ART. It may be that ART is commenced when the CD4+ count is already too low, and with more severe immunocompromise. A CD4+ count $>200$ cells/ $\mu \mathrm{l}$ was associated with lesser grade of disease at follow-up Pap smear, but was only statistically significant for CD4+ counts of 200 - 350 cells/ $\mu$ l. Incomplete excision of the lesion reduced the chance of a lesser grade of disease on the repeat Pap smear. However, the association failed to reach significance when disease was present only at the endocervical margin.

\section{Lost to follow-up}

The loss to follow-up was extremely high, which is often the case when doing retrospective research in a clinical setting, as opposed to the research situation as seen in other studies on the treatment of CIN. ${ }^{11}$ We therefore compared the women who attended the followup clinic with those who had not. Median CD4+ count was lower in women who did not attend (177 (IQR 89 - 300) v. 203 (IQR 125 - 340) $(p=0.01))$. Women who used ART $(p=0.04)$ were more likely to be lost to follow-up (49.08\% v. $40.96 \%)$. This may be because women who did not use ART were ill and therefore did not attend follow-up or that they had less contact with health professionals. Therefore, these results might not reflect the true picture regarding the association between these factors and disease at follow-up.

\section{Discussion}

Abnormal cytology findings at follow-up in this HIV-infected population of women occurred in $65.03 \%$, which is comparable to other studies. ${ }^{6,12}$ However, none of those studies evaluated the grade of the disease after treatment. Our study showed an encouragingly high rate of regression of the CIN (72.88\%).

The relationship between the use of ART and cervical neoplasia is not well established. Some studies have shown that ART promotes the regression of the disease, and others found no influence of ART on regression of cervical neoplasia. ${ }^{6}$ In our study, patients who used ART had a statistically significantly lower chance of a lesser grade of disease than patients who did not use ART. However, the length of time on ART and CD4+ count at initiation could be confounders that we could not control for. Furthermore, we did not have serial CD4+ counts, which might also have affected our results. To properly assess the effect of ART, we need longitudinal data on duration of use, viral loads, and changes in CD4+ counts.

That the presence of disease at any margin was associated with a worse outcome is not surprising, and treatment should aim at complete excision. However, the lesions are often larger in HIVinfected women and often associated with satellite lesions. Lesion size in this group was not recorded but, in a similar group of HIVnegative women at this clinic, complete excision was achieved in
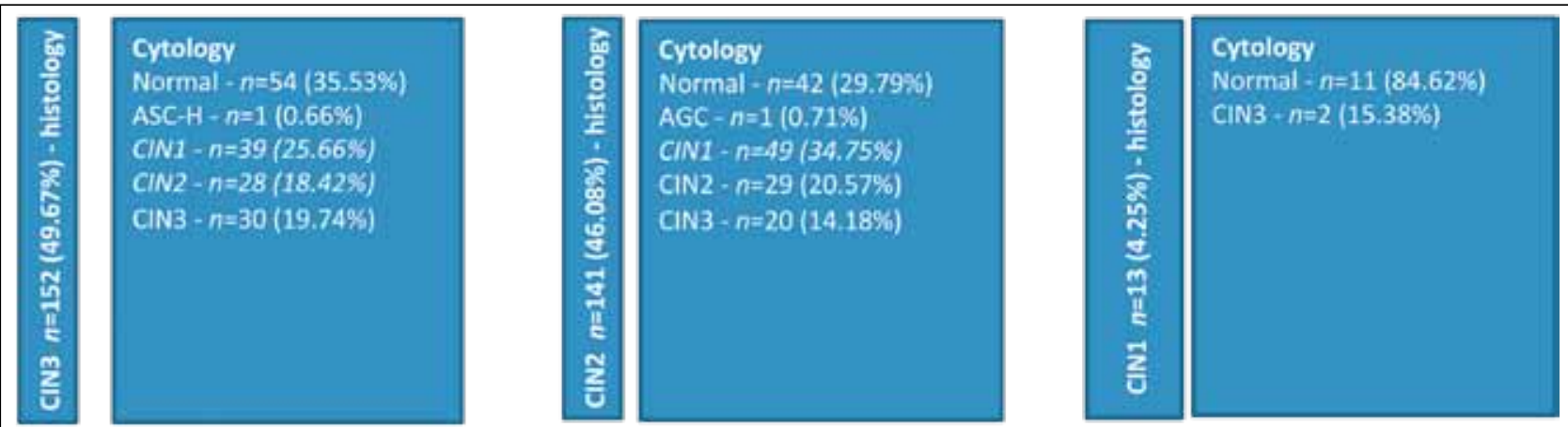

Fig. 2. Histology results at treatment and Pap smear at follow-up. The Pap smear findings shown in italics are what was considered a lesser grade of disease or normal $(72.88 \%)$. 
49.4\% (unpublished data). Complete excision in this group of women was achieved in $38.33 \%$. Note that these women might have been younger and desiring pregnancy, so extensive excision might not always be appropriate.

A limitation of this research is that it was carried out in a clinical setting and therefore associated with a large loss to followup. Other risk factor information such as smoking, sexual history and duration of contraception use was not collected. A further limitation was that cytology results at follow-up were compared with histology results obtained initially at LLETZ to determine whether there was a change in the grade of the lesion. Despite a study at this clinic in 2006 finding that the correlation between HSIL on cytology and CIN2/CIN3 on histology was $78.96 \%$ (13), ${ }^{13}$ the comparison would best be made by comparing histology with histology.

\section{Conclusion}

Surgical treatment of CIN in HIV-infected women is associated with a high persistence of disease; however, this is of a lesser grade. Further study of these women with serial CD4+ counts, viral loads, treatment effect and HPV typing is warranted.

\section{References}

1. NHLS. National Cancer Registry. http://www.nhls.ac.za/index.html (accessed 27 June 2010).

2. AIDS and HIV information from AVERT. www.avert.org/aidssouthafrica.htm (accessed 26 February 2008). 3. Parham G, Sahasrabuddhe V, Mwanahamuntu M, Shepherd B, Hicks M, Stringer E. Prevalence and predictors of squamous intraepithelial lesions of the cervix in HIV-infected women in Lusaka, Zambia. Gynaecol Oncol 2006;103:1017-1022.

4. Peto J, Gilham C, Fletcher O, Matthews F. The cervical cancer epidemic that screening has prevented in the UK. Lancet 2004;364:249-256.

5. Soutter W, Sasieni P, Panoskaltsis T. Long-term risk of invasive cervical cancer after treatment of squamous cervical intraepithelial neoplasia. Int J Cancer 2006;118:2048-2055.

6. Heard I, Potard V, Foulot H, Chapron C, Costagliola D, Kazatchkine M. High rate of recurrence of 6. Heard I, Potard V, Foulot $\mathrm{H}$, Chapron C, Costagliola D, Kazatchkine M. High rate of recurrence of
cervical intraepithelial neoplasia after surgery in HIV-positive women. J Acquir Immune Defic Syndr 2005;39(4):412-418.

7. Adam Y, van Gelderen CJ, de Bruyn G, McIntyre JA, Turton DA, Martinson NA. Predictors of persistent cytologic abnormalities after treatment of cervical intraepithelial neoplasia in Soweto, South Africa: A cohort study in a HIV high prevalence population. BMC Cancer (Research Article) 2008;8(211).

8. Solomon D, Davey D, Kurman R, et al. The 2001 Bethesda System.Terminology for reporting results of cervical cytology. JAMA 2002;287(16):2114-2119.

9. Das S, Elias A. Diagnosis and treatment of cervical intra-epithelial neoplasia in a single visit. Aust NZJ Obstet Gynaecol 1998;38(3):246-250.

10. Xi L, Kiviat N, Wheeler C, Kreimer A, Ho J, Koutsky L. Risk of cervical intraepithelial neoplasia grade 2 or 3 after loop electrosurgical excision procedure associated with human papillomavirus type 16 variants. J Infect Dis 2007;195(9):1340-1344.

11. Spitzer M, Chernys A, Seltzer V. The use of large loop excision of the transformation zone in an innercity population. Obstet Gynecol 1993;82:731-735.

12. Fruchter R, Maiman M, Sedlis A, Bartley L, Camilien L, Arrastia C. Multiple recurrences of cervical Fruchter R, Maiman M, Sedlis A, Bartley L, Camilien L, Arrastia C. Multiple recurrences of cervical
intraepithelial neoplasia in women with the human immunodeficiency virus. Obstet Gynecol 1996;87:338-344.

13. Adam Y, van Gelderen C, Newell K. Look and Lettz: a South African experience. S Afr Med J 2008;97(2):112-122.

Accepted 22 June 2012. 